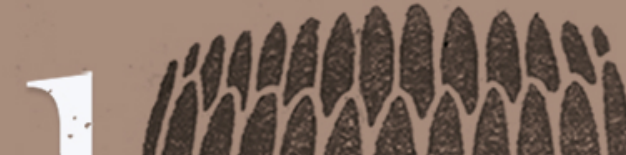



vol. 4, Aum?2

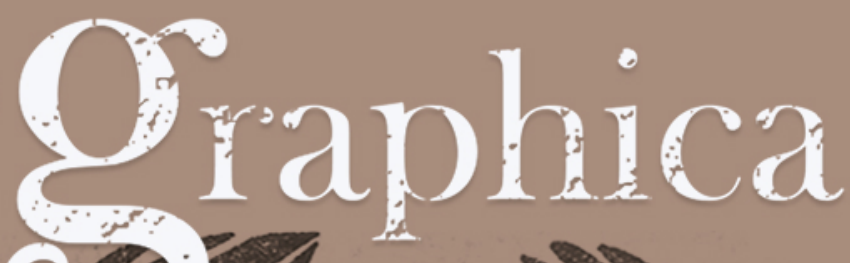

segubdo-semestre 2021 s.

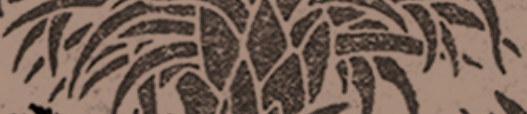
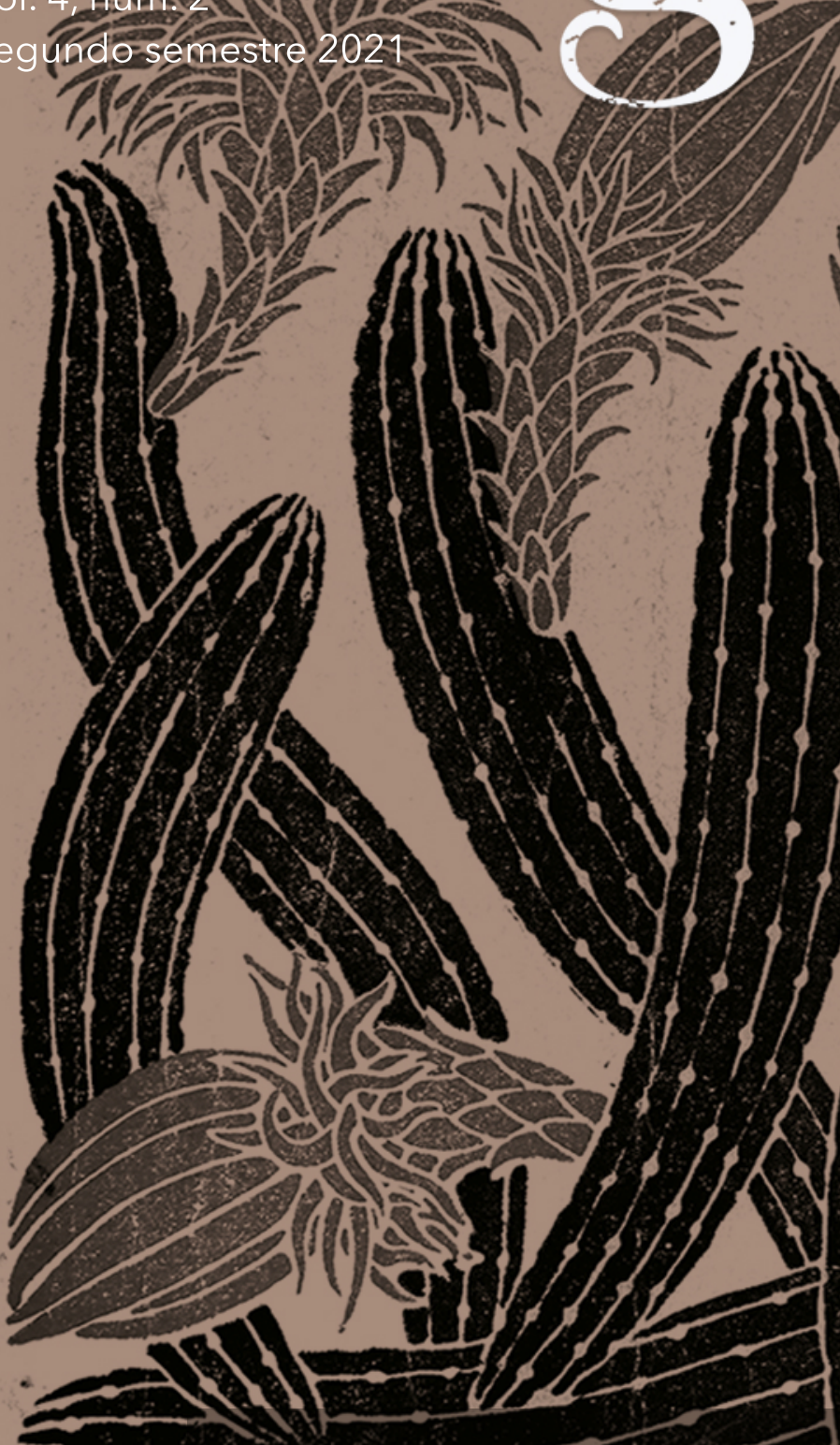

ISSN $2594-178 X$
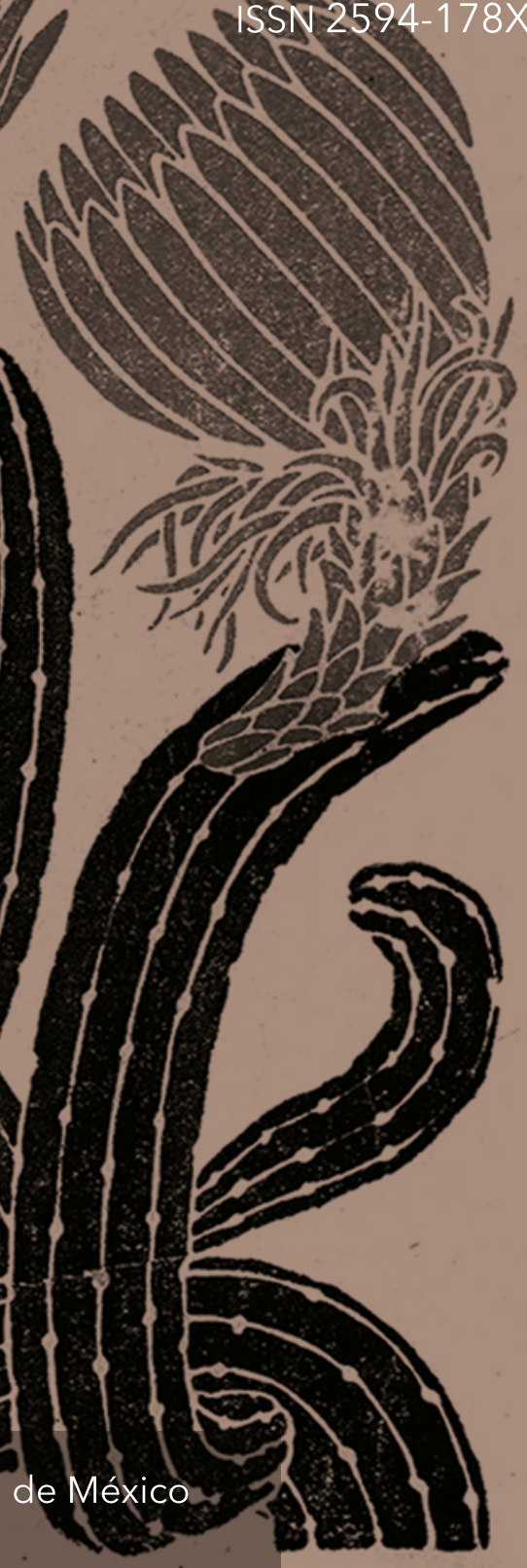


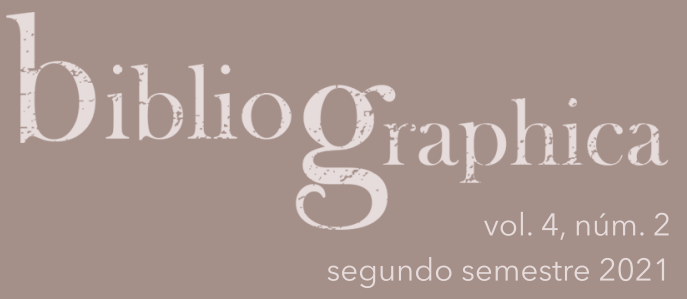

\section{Propuesta de una tipología de los manuscritos novohispanos en lenguas indígenas de la Biblioteca Nacional de México}

Proposal for a Typology of Colonial

Manuscripts in Indigenous Languages

Held in the Biblioteca Nacional de México

\section{Tesiu Rosas Xelhuantzi}

Universidad Nacional Autónoma de México, Instituto de Investigaciones Bibliográficas,

Ciudad de México. México

tesiurosas@gmail.com

ORCID: http://orcid.org/0000-0002-8557-2977

Recepción: 13.04.2021 / Aceptación: 24.06.2021

DOI: https://doi.org/10.22201/iib.2594178xe.2021.2.108 
Resumen A partir de la revisión catalográfica y el estudio material de los documentos, este artículo delinea los ejes temáticos presentes en el corpus de manuscritos novohispanos en lenguas indígenas conservados en la Biblioteca Nacional de México. Derivada de un análisis comparativo, se propone una clasificación tipológica que considera cuatro divisiones denominadas géneros documentales. La conclusión a la que llega el planteamiento es que el abordaje de la colección como objeto de estudio permite rutas de investigación complementarias a los análisis individuales de los manuscritos.

Palabras Manuscritos; lenguas indígenas; clasificación tipológica; Nueva España; siglos clave XVI-XVIII.

Abstract Derived from a catalogs review and the material study of the documents, this paper outlines the thematic axes found in the corpus of colonial manuscripts in indigenous languages preserved by the Biblioteca Nacional de México (National Library of Mexico). Based on a comparative analysis, this article proposes a typological classification of four divisions called documentary genres. The conclusion is that approaching the collection as an object of study allows ancillary research paths to the individual analysis of the manuscripts.

Keywords Manuscripts; indigenous languages; typological classification; New Spain; 16th18 th centuries. 


\section{Introducción ${ }^{1}$}

La Biblioteca Nacional de México (BNM) resguarda un conjunto de manuscritos novohispanos en lenguas indígenas que conforman un importante legado histórico y muestran el contacto inicial entre el mundo mesoamericano y el europeo. Algunos de estos ejemplares fueron utilizados durante el siglo XVI en el proceso de evangelización de los pueblos originarios. Entre los investigadores que resaltan la importancia de esta colección se encuentra Ascensión Hernández Triviño, quien plantea lo siguiente: "la Biblioteca Nacional de México posee cuantiosos documentos y varias piezas maestras de manuscritos en náhuatl generados en los tempranos focos creadores, además de otros muchos de los siglos XVII y XVIII [...]. Puede decirse que la Biblioteca Nacional es uno de los mayores repositorios en lengua náhuatl existentes en el país".?

El primer acercamiento especializado a la colección de manuscritos en lenguas indígenas de la BNM fue realizado en 1945 por Ángel María Garibay Kintana, cuando desarrollaba la investigación histórica para su obra Historia de la literatura náhuatl. ${ }^{3}$ Las notas que tomó Garibay fueron presentadas en forma de guía bibliográfica y publicadas en 1966 con el título "Manuscritos en lengua náhuatl de la Biblioteca Nacional de México".4

Esta guía de manuscritos adquirió relevancia por su papel pionero, así como por los comentarios eruditos que el autor elaboró sobre varias de las obras registradas. Sin embargo, presenta dos limitantes importantes respecto al conjunto documental: la primera es que sólo incorporó 21 ejemplares, que serían menos de un tercio del total de los manuscritos; la segunda es que solamente se enfocó en el náhuatl y no recuperó otros idiomas indígenas. La lengua náhuatl se encuentra registrada en aproximadamente $80 \%$ de los ejemplares del corpus, mientras que en el porcentaje restante aparecen diferentes lenguas

\footnotetext{
${ }^{1}$ La investigación realizada para este artículo fue apoyada por el Conacyt a través del Programa de Estancias Posdoctorales por México, bajo la dirección de la doctora Marina Garone Gravier, a quien agradezco encarecidamente su valiosa asesoría.

2Ascensión Hernández Triviño, "La lengua náhuatl en los archivos y bibliotecas de México", en Lenguas y escrituras en los acervos bibliohemerográficos. Experiencias en el estudio de la tradición clásica, indígena y contemporánea, coord. de Marina Garone y Salvador Reyes Equiguas (México: UNAM, 2019), 76.

${ }^{3}$ Ángel María Garibay Kintana, Historia de la literatura náhuatl (México: Porrúa, 1953).

${ }^{4}$ Ángel María Garibay Kintana, "Manuscritos en lengua náhuatl de la Biblioteca Nacional de México", Boletín de la Biblioteca Nacional de México 17, 2a. época, núms. 1-2 (enerojunio de 1966): 5-19.
} 
indígenas mesoamericanas y norteamericanas, tal como ejemplifica Miguel León-Portilla: "Y no se limita el tesoro de los manuscritos indígenas a los textos en náhuatl. Mencionaré al menos la existencia de otros en otomí, maya, mixteco, ópata, totonaco, así como en lenguas habladas en las costas septentrionales de América". ${ }^{5}$ La publicación de Garibay se concentró en los manuscritos en náhuatl debido a la ruta de investigación histórica que estaba siguiendo; recordemos que no buscaba elaborar una guía bibliográfica, sino que inicialmente fueron apuntes personales para su obra.

El segundo acercamiento fue elaborado por Roberto Moreno de los Arcos, quien continuó el trabajo iniciado por Garibay y lo complementó con una metodología que unificó criterios de descripción, lo cual le permitió registrar la totalidad de manuscritos en lenguas indígenas que la BNM poseía en 1966, consistente en 50 manuscritos en náhuatl y 14 en otras lenguas originarias. El resultado fue la "Guía de las obras en lenguas indígenas existentes en la Biblioteca Nacional", ${ }^{6}$ que en la actualidad constituye la principal referencia para acercarnos al corpus. Sin embargo, también tiene dos limitantes de relevancia, relacionadas con la cantidad y la cualidad de los manuscritos.

La primera está vinculada al total de ejemplares que componen el corpus, que en la actualidad es mayor a los 64 manuscritos registrados por Moreno de los Arcos en 1966; es justo aclarar que no se trata de un error del autor, sino que la guía ha quedado rebasada con el tiempo porque la BNM ha adquirido más manuscritos en lenguas indígenas durante los últimos 50 años, los cuales no aparecen en la publicación de Moreno.

La segunda limitante de esta guía es que no quedó completa la identificación de todas las lenguas indígenas diferentes al náhuatl. Moreno alcanzó a postular algunas propuestas de identificación en ciertos casos, pero en otros se limitó a registrar el nombre de la lengua, de acuerdo a la nomenclatura novohispana utilizada en el manuscrito. La implicación que ello conlleva es que mientras no se termine el trabajo de identificación lingüística no es posible hacer una lista con la cantidad exacta de lenguas amerindias que aparecen en el corpus.

Las guías de Garibay y Moreno de los Arcos constituyen un punto de referencia para el estudio de los manuscritos en lenguas indígenas. Los límites que

\footnotetext{
${ }^{5}$ Miguel León-Portilla, "El tesoro de los manuscritos indígenas", en La Biblioteca Nacional, triunfo de la República, coord. de Vicente Quirarte (México: UNAM, 2006), 81.

${ }^{6}$ Roberto Moreno de los Arcos, "Guía de las obras en lenguas indígenas existentes en la Biblioteca Nacional", Boletín de la Biblioteca Nacional de México 17, 2a. época, núms. 1-2 (enero-junio de 1966): 21-117.
} 
alcanzaron sobre la cantidad y la cualidad de los ejemplares se convierten en rutas abiertas de investigación que pueden ser abordadas con nuevos enfoques y alcances. Si bien ya existen estudios recientes que han logrado profundizar en la comprensión de importantes obras del acervo como los Cantares mexicanos, ${ }^{7}$ en cambio no se ha desarrollado el estudio de la colección en su conjunto.

En este artículo se elabora una aproximación al conjunto de los manuscritos novohispanos en lenguas indígenas conservados en la BNM. Parte de la premisa de que se requiere afinar la comprensión de ese corpus de manuscritos como objeto de estudio. En esa dirección, plantea una propuesta tipológica que busca identificar los géneros documentales asociados de forma específica al corpus. La propuesta deriva en la clasificación de cuatro géneros documentales que serán brevemente planteados y ejemplificados.

\section{Antecedentes de clasificación}

Los manuscritos de tradición indígena pueden clasificarse con diferentes criterios, dependiendo de los objetivos que se busquen y de las disciplinas desde las que sean abordados. Algunos ejemplos simplificados de márgenes de acción con diferentes criterios de clasificación son los siguientes: cronológico, regional, cultural, escriturario y material.

Con un criterio cronológico sería posible separar la época prehispánica de la colonial y ordenar los manuscritos por fecha en orden creciente; en cambio, con un criterio cultural sería posible asociar manuscritos de acuerdo con su tradición cultural y lingüística, lo cual podría traspasar la división temporal entre lo prehispánico y lo colonial.

El criterio regional agruparía los manuscritos por zonas geográficas, lo que podría llegar a generar coincidencias con la agrupación cultural. Otro enfoque sería el criterio escriturario, que enfatizaría los sistemas de escritura con los cuales se registran los manuscritos y que generalmente realizaría una distinción entre la escritura jeroglífica y la alfabética; en forma muy cercana, se encontraría el énfasis en la iconografía que suele manifestarse en los manuscritos con modelos estéticos, que pueden inclinarse hacia lo mesoamericano o hacia lo medieval y renacentista, es decir, con un carácter híbrido.

Un ejemplo más sería el criterio basado en el formato del manuscrito y en su materialidad, con lo que se pondría mayor atención en el tamaño y sopor-

\footnotetext{
${ }^{7}$ Miguel León-Portilla, ed., Cantares mexicanos (México: UNAM, 2019).
} 
te de los manuscritos, así como en la comprensión de su elaboración material. Con los ejemplos mostrados se intenta explicar que no existe una forma única de clasificar los manuscritos de tradición indígena, sino que depende de los objetivos por los que se busca su clasificación, al igual que de la metodología disciplinaria utilizada.

A continuación, vamos a detenernos en la propuesta de clasificación tipológica elaborada por John B. Glass. ${ }^{8}$ Este autor se concentra en manuscritos pictográficos de tradición indígena y toma como eje de su clasificación el contenido temático. La tipología de Glass logra una fácil agrupación con ciertos tipos de manuscritos, como los rituales-calendáricos y los económicos, que incluso cuentan con subdivisiones; sin embargo, no resulta tan clara al agrupar los etnográficos o históricos, pues en amplio sentido todos los manuscritos son históricos y muestran rasgos de una cultura.

En su propuesta aparecen tres rubros que no constituyen un tipo, pero que resultan estrategias interesantes para problemas específicos. El primero es el de miscelánea, que incluye ítems que no lograron corresponder de manera precisa a los tipos delineados. El segundo rubro, denominado sin-clasificación, se refiere a los manuscritos que no alcanzó a clasificar por falta de información o acceso a reproducciones fotográficas de los ejemplares. Finalmente incluye un rubro que puede traducirse como no-disponible, en el que enlista ejemplares conocidos a través de referencias bibliográficas, pero que al buscarlos no fueron localizados y su paradero es desconocido.

En la siguiente tabla se aprecia la clasificación tipológica elaborada por Glass.

La propuesta de Glass es un buen punto de partida para atender la clasificación especializada de un corpus de manuscritos de tradición indígena, pero no debe considerarse completa. El mismo autor definió los catecismos testerianos como una clase de manuscritos pictográficos y, aunque tiene un apartado sobre ellos en el mismo volumen, ${ }^{9}$ no los incorporó en su tipología. Asimismo, quedó fuera el género de manuscritos Techialoyan, recuperado por Donald Robertson también en el mismo volumen. ${ }^{10}$ A pesar de ello, el planteamiento de Glass tiene

\footnotetext{
${ }^{8}$ John B. Glass, "A Survey of Native Middle American Pictorial Manuscripts", en Handbook of Middle American Indians. Guide to Ethnohistorical Sources, vol. 14, ed. de Howard Cline (Austin: University of Texas Press, 1975), 3-80.

9 John B. Glass, "A Census of Middle American Testerian Manuscripts", en Handbook of Middle American Indians, 281-296.

${ }^{10}$ Donald Robertson, "Techialoyan Manuscripts and Paintings, with a Catalog", en Handbook of Middle American Indians, 253-280.
} 
el mérito de haber perfilado un debate -que aún hoy sigue abierto- sobre las formas y finalidades de clasificar y definir los manuscritos de tradición indígena.

\begin{tabular}{|c|c|}
\hline \multicolumn{2}{|c|}{$\begin{array}{l}\text { Typological Classification Used in the Census } \\
\text { of Traditional Pictorial Manuscripts }\end{array}$} \\
\hline Ritual-calendrical & \\
\hline & 260-day almanacs \\
\hline & 18-month calendars \\
\hline & Calendar wheels \\
\hline \multicolumn{2}{|l|}{ Historical } \\
\hline \multicolumn{2}{|l|}{ Genealogical } \\
\hline \multicolumn{2}{|l|}{ Cartographic } \\
\hline \multicolumn{2}{|l|}{ Cartographic-historical } \\
\hline \multicolumn{2}{|l|}{ Economic } \\
\hline & Cadastral \\
\hline & Census \\
\hline & Financial records \\
\hline & Property plans \\
\hline & Tribute \\
\hline \multicolumn{2}{|l|}{ Ethnographic } \\
\hline \multicolumn{2}{|l|}{ Miscellaneous } \\
\hline & Litigative \\
\hline & Natural History \\
\hline Unclassified & \\
\hline Unavailable & \\
\hline
\end{tabular}

Clasificación tipológica de manuscritos pictográficos de tradición indígena, elaborada por Glass. Fuente: John B. Glass, "A Survey of Native Middle American Pictorial Manuscripts", en Handbook of Middle American Indians. Guide to Ethnohistorical Sources, vol. 14, ed. de Howard Cline (Austin: University of Texas Press, 1975), 28.

En la tipología que aquí se propone recuperamos el planteamiento de Glass en el aspecto de clasificar por temáticas, aunque no se siguieron las mismas divisiones. Después de realizar un análisis comparativo dentro de la colección de manuscritos conservados en la BNM, se alcanzaron a identificar cuatro ejes temáticos que fueron considerados como géneros documentales dentro del corpus: a) Lingüístico; b) Religioso; c) Legal; d) Códices pictográficos. También se identificó que dentro de estos ejes temáticos aparecen modelos textuales que definen el tipo de cada manuscrito. De esta manera, se logró establecer una propuesta de clasificación documental del corpus que en su nivel general ubica a los géneros, y en su nivel particular a los tipos. A continuación son descritos 
cada uno de los géneros y tipos documentales propuestos, con ejemplos representativos que forman parte del corpus analizado.

\section{Género lingüístico}

Está integrado principalmente por dos tipos de obras conocidas como artes y vocabularios. La fórmula Arte + Vocabulario constituyó un modelo inspirado en Antonio de Nebrija que fue ampliamente recuperado no sólo en Nueva España, sino también en todas las colonias hispánicas. Aunque no siempre se siguió este modelo y se elaboraron artes o vocabularios de forma aislada, en general fue considerada como una aspiración la elaboración de ambas obras complementarias. ${ }^{11}$

El MS 1494 es un buen ejemplo de este género lingüístico: contiene en su primera parte el Arte de la lengua Teguima elaborado por Natal Lombardo y en su segunda parte se complementa con el Vocabulario de la lengua Ore.

Otro ejemplo es el MS 1497, donde encontramos el Diccionario de Otomí, elaborado por un autor desconocido a mediados del siglo XVII.

Por su parte, el MS 1474 muestra que incluso en la última fase de Nueva España aún se utilizaba el modelo de Nebrija, como se puede encontrar en la Gramática de la lengua totonaca elaborada por José Zambrano en el siglo XVIII.



Imagen 1. Arte de la lengua Teguima de Natal Lombardo, MS 1494, f. 1 r.

\footnotetext{
${ }_{11}$ Sobre el modelo metodológico Arte + Vocabulario en el contexto colonial de las Américas, véase Tesiu Rosas Xelhuantzi, "Colonización lingüística y subversión decolonial nahua en el siglo XVI", NuestrAmérica 6, núm. 11 (enero-junio de 2018): 72-89.
} 


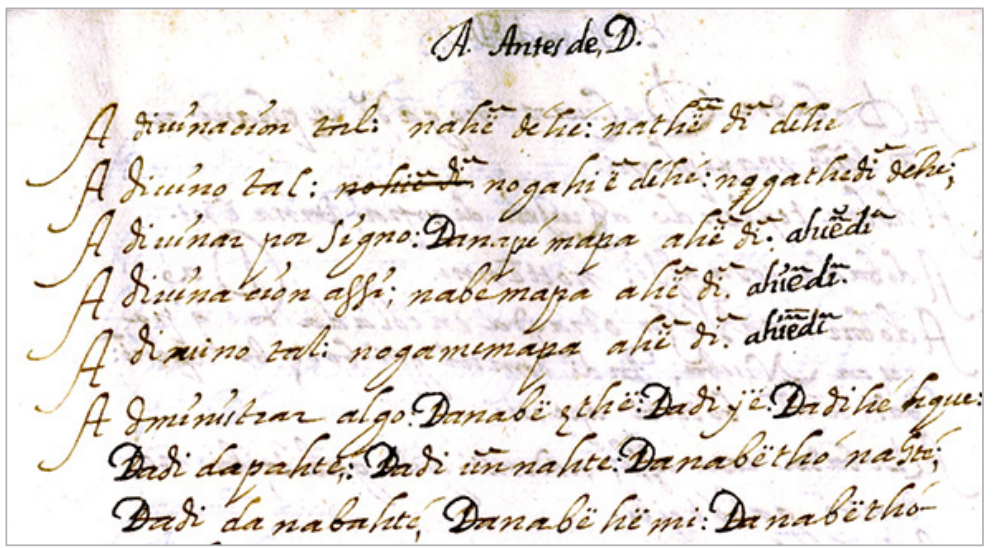

Al final del periodo novohispano apareció una vertiente que se separó de la fórmula Arte + Vocabulario asociada a un contexto religioso. Mientras que en los siglos XVI y XVII los vocabularios en lenguas indígenas estaban vinculados principalmente al trabajo de los frailes, en el siglo XVIII encontramos vocabularios relacionados con la exploración del norte de Nueva España y los territorios en disputa.

El objetivo de estos vocabularios se concentró en la comunicación con las poblaciones originarias en un contexto de exploración, por lo que se elaboraron listas de vocablos de las lenguas locales para facilitar el intercambio comercial, las negociaciones, el ejercicio de la administración colonial e incluso para complementar los registros científicos que ya incluían las exploraciones.

Tal es el caso del MS 1759, que registra diversos aspectos geográficos, naturales y sociales de la isla de Nutka, localizada en lo que actualmente es Vancouver, Canadá, pero que en el siglo XVIII era un área en disputa entre el imperio español y el inglés. Este manuscrito incluye un vocabulario de la lengua Nutka, cuyo objetivo comunicativo estuvo asociado al registro de la exploración, como puede leerse en un fragmento: "Nuestra mansion de mas de 4 meses en aquella Ysla me facilitó el informarme de varias costumbres de sus Naturales, de cuya Religion y sistema de gobierno creo haber sido el primero que há recabado las noticias posibles despues de haber aprehendido de su idioma lo preciso para mantener con ellos algunas conversaciones". ${ }^{12}$

\footnotetext{
${ }^{12}$ MS 1759, f. 4r.
} 
Con el mismo sentido práctico comunicativo fueron elaborados los vocabularios de Nutka, Inuit y Aleutiano que se encuentran en el MS 1683, titulado "Reconocimiento de los quatro establecimientos que el Ymperio Ruso ha formado al Norte de la California". Dentro del manuscrito, que aborda diferentes asuntos sobre la exploración, está la traducción al castellano de pequeños vocabularios elaborados por el capitán inglés James Cook entre 1788 y 1789, cuando exploraba la costa noroccidental de América. El título de la sección que contiene los vocabularios nos muestra su objetivo:

Vocabularios del Lenguaje, Idioma, o dialecto de los Indios de las Costas septentrionales y occidentales de America en los 49-53-60 y 64 grados de Latitud norte. Para el uso, inteligencia y govierno de los Encargados de la nueva Exploracion a aquellas Alturas en virtud de la Real Orden de 26 de Enero de 1787. Deducido todo de la practicada por el Ingles Jacobo Cook en su tercer viage al descubrimiento de el globo. ${ }^{13}$

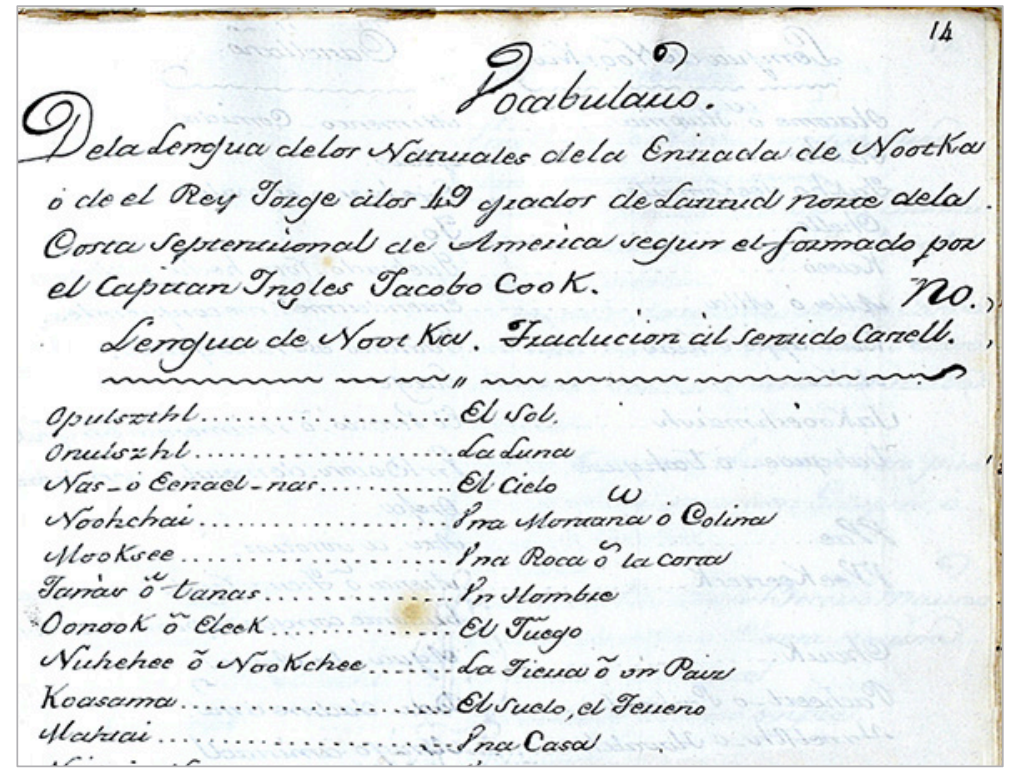

Imagen 3. Vocabulario de Nutka, MS 1683, f. 14r.

\footnotetext{
${ }^{13}$ MS 1683, f. 13 r.
} 


\section{Género religioso}

El género religioso es el más abundante dentro del corpus de manuscritos en lenguas indígenas, así como el más complejo en cuanto a la variedad de temáticas desarrolladas. Es posible identificar al menos a dos lectores-objetivo: por una parte, hay obras destinadas principalmente al auxilio de los frailes en su labor de conversión y consolidación de la fe cristiana; por otra, están las obras dirigidas directamente a los indígenas, para el fortalecimiento de sus prácticas religiosas cotidianas.

Entre los tipos más desarrollados dentro del corpus está el de los sermones, que puede asociarse a un microcorpus de 11 manuscritos que abarcan del MS 1480 hasta el MS 1488, además del MS 1493 y el MS 10275. En estos manuscritos en lengua náhuatl se puede encontrar la intención de servir como un apoyo directo al religioso en su labor de prédica, por lo que suelen estar ordenados de acuerdo al calendario litúrgico, y dispuestos para ser leídos en misa. El contenido puede partir de pasajes bíblicos, a los cuales sigue una explicación extendida que tiene el reto de hacer comprensibles las premisas del cristianismo para un público indígena.

El tipo de evangeliarios está vinculado de forma cercana al de los sermones. En los evangeliarios encontramos traducciones de secciones de la Biblia a la lengua indígena; los sermonarios podían retomar estas traducciones para ser comentadas y desarrolladas con una exposición en forma de sermón, por lo cual están vinculados ambos tipos documentales, sin embargo, es posible encontrar de forma independiente los evangeliarios en manuscritos como el MS 1492 y el MS 1478.

Cabe mencionar que en el MS 1482 se encuentran ocho fojas sueltas que fueron arrancadas de distintos evangeliarios en náhuatl y que son diferentes al sermonario en náhuatl que está en la misma clasificación.

El tipo de santorales también se relaciona de forma cercana con los sermonarios, que en ocasiones desarrollan sermones para abordar la vida de los santos. Aún así, es posible encontrar la influencia de un molde literario independiente relacionado con los relatos hagiográficos medievales encontrados en los flos sanctorum; los santorales en lenguas indígenas parecen recuperar este molde, con una adaptación pensada para un público indígena. Algunos ejemplos de santorales en lenguas indígenas que tienen influencia de un modelo hagiográfico serían los manuscritos MS 1475 y MS 1476. 
Dominica quadagefimale/inquin quagefima the. silinguis frominum loguar stangeloy. ef.

$\int_{n}$ ntfacuiloli onffamantfi mopia ȳricatfalficpac yniccentlamantfi yntfaixis

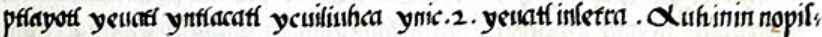
uate uslanguimati ynican antfaca cayausenuth amquipiaya ynamotfacuilo $\dot{y}$

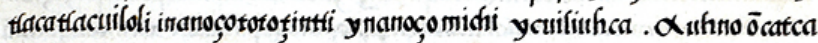
amomauh initeof antlalmamiquia ynimcaquaficatca ynäquichiuaya, tel amouefix quidt anquimatia caçanited antflaftamiquia . Xuhinin caanquita

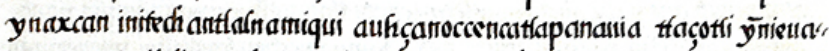
tr letta tracuidoli cauel oncanmifona inquenami tfotofi, aufrinflajxiptlaioff anquita yntacatiacuilofi anquimati caonca ynima ymicgei çanaud tiafit" quiya atuel menemi noonca yniten yninacaz ymixtelolo çanauel tlatoaaud



Imagen 4. Sermonario Sahagún-Escalona, MS 1482, f. 47r.

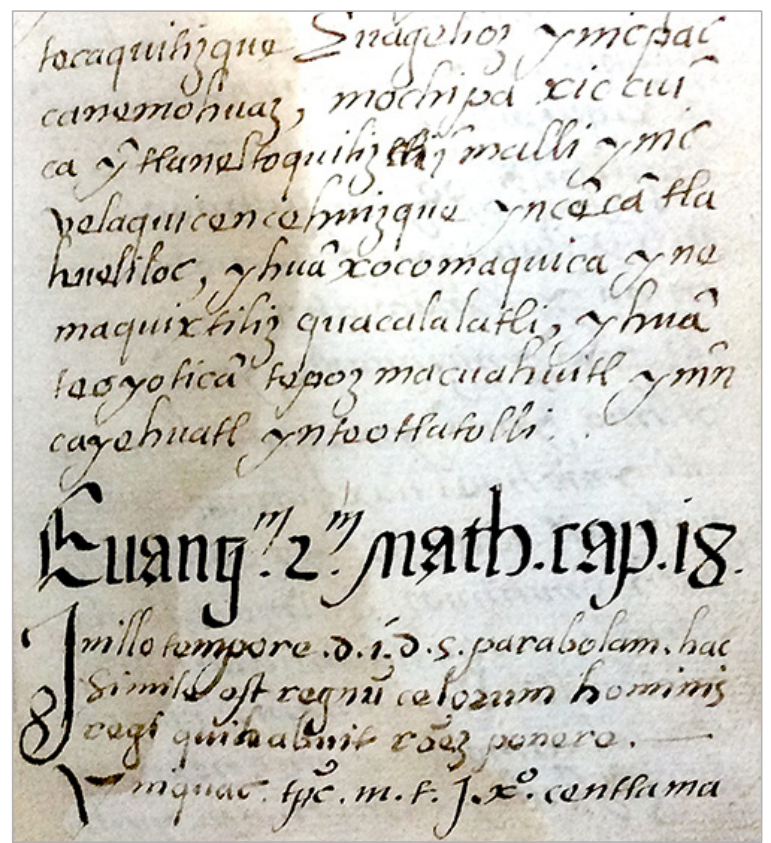

Imagen 5. Epistolario y Evangeliario, MS 1478, f. 127 r. 
El tipo de miscelánea religiosa contiene un conjunto de obras diversas y complejas, algunas de ellas desarrolladas dentro del contexto de evangelización del siglo XVI. La propuesta tipológica considera la necesidad de un espacio en el que entren ejemplares difíciles de clasificar de forma homogénea, ya que pueden abordar diversas temáticas, con enfoques que van desde lo inquisitorial hasta lo experimental.

Por ejemplo, el MS 1488 tiene en la primera parte una sección de sermones, varios de ellos relativos a santos, y en la segunda se encuentra el Tratado sobre los siete pecados capitales y el Tratado sobre el demonio, elaborados en náhuatl por fray Andrés de Olmos; por otro lado, en el MS 1477 están los Colloquios de la paz y tranquilidad Christiana en lengua mexicana de fray Juan de Gaona, seguidos de otros textos dirigidos a la formación de príncipes indígenas, cuya elaboración y compilación posiblemente está ligada al trabajo de los propios intelectuales nahuas. Este manuscrito tiene el título facticio de Miscelánea sagrada, el cual refleja el sentido amplio que en este artículo se le asigna al tipo de miscelánea religiosa.

Otro ejemplo relevante de este tipo documental es el MS 1628 conocido como Cantares mexicanos, el cual ha sido considerado una obra de literatura náhuatl. Si bien es cierto que pueden asociarse con la literatura las fábulas de Esopo y los cantos en mexicano que han sido tomados para el título facticio, el conjunto del manuscrito se ubica dentro de un contexto religioso. Además, el ejemplar presenta una diversidad de temas religiosos tales como sermones, relatos de vida de santos con influencia de santorales y el Arte adivinatoria de los mexicanos, en el que se describen los signos calendáricos y las ceremonias asociadas a ellos. Los manuscritos del tipo de miscelánea religiosa muestran la complejidad y diversidad de la producción textual religiosa, elaborada principalmente en el siglo XVI.

En contraste, el tipo de oraciones y rosarios representa el perfil práctico y simplificado que se destinó a los creyentes indígenas al final del periodo novohispano. Estos ejemplares conforman un micro-corpus de nueve manuscritos del siglo XVIII colocados de la clasificación 40/7/1 a la 40/7/7, además del MS 566 y el MS 858. Es posible perfilar, en términos amplios, que el contenido textual del género religioso tiende a ser más complejo en manuscritos de los siglos XVI y XVII, por ejemplo en sermones, tratados religiosos y traducciones bíblicas, mientras que al final del periodo novohispano se privilegian las obras prácticas y sencillas, como libros de oraciones y rosarios. 


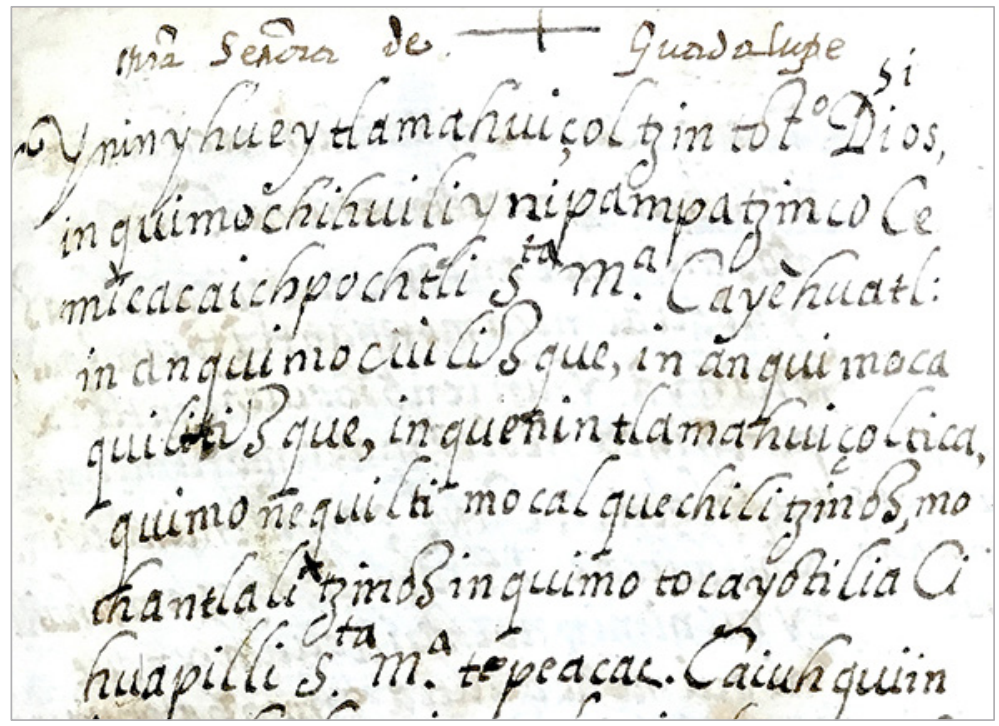

Imagen 6. Santoral en mexicano, MS 1475, f. 51 r.

- DECOMO ELDEMONIODE 4 Jinc jicap' ypamitor ŷquem yevaft

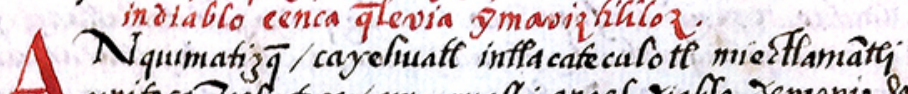
- ynifoca. icly toca amo qualy angel riabto Semonio Sa 1 Lhan f. Xro mielpa anquimocaquitia ynovalfuste ynil fulical y pampa ì cenca huey yoniffatfacul ynompa oquithich ynomopouf yno affamal ocuecuenot, nima amo oquiffaca mafiznec ynicelfin nelli sios ynompa oquiyocox oquipic oquishing oquiffacafil;: canixero yoprac omoquetr yoâ pâve" teiznequia yyan po furizequia cenca ycomonimito omocue ponaffi quimonene furihinequia ynipafnemoani y toque $\hat{y}$

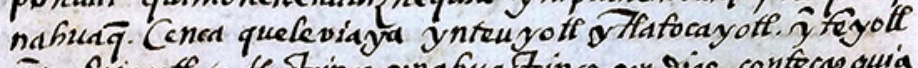
Imasizute y frothince ynafualuinco yn Dios conferaqua ynsinblo ynipeti onipal onnicquinamiquitnequid. brasi

Imagen 7. Tratado sobre el Demonio de Andrés de Olmos, MS 1488, f. 394v. 

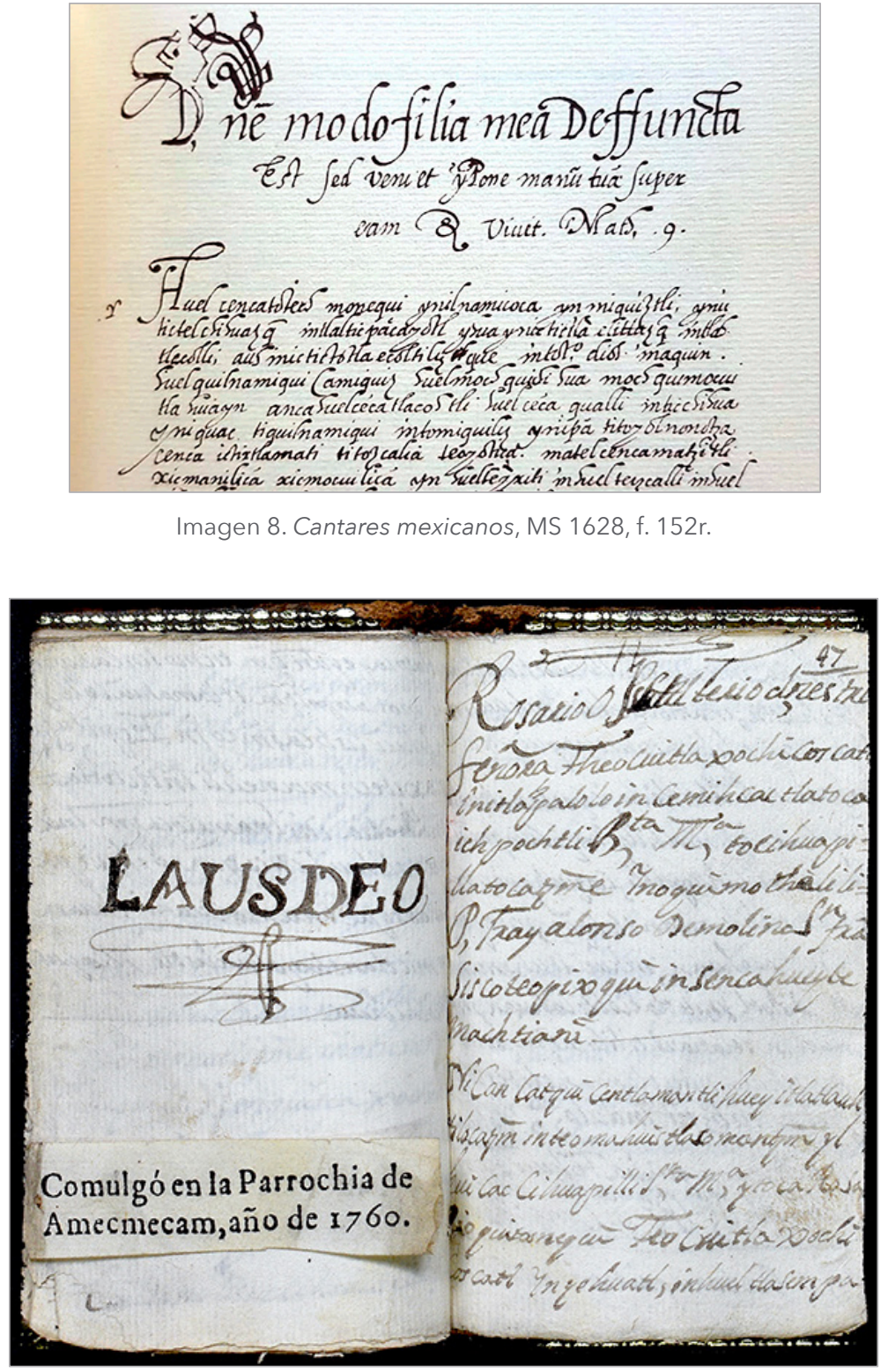

Imagen 9. Rosario de la virgen María, MS 566, f. 47r. 


\section{Género legal}

Pasando a otro género documental, es viable agrupar los manuscritos en lenguas indígenas con un perfil legal asociado a las prácticas administrativas. Al revisar ejemplares del género legal, se identificaron los tipos documentales de testamentos, procesos y peticiones.

En el tipo de testamentos se encuentran transcripciones textuales de la voluntad del testador, que declaraba en su lengua originaria. Un ejemplo lo constituye el MS 1233, un expediente de documentos legales del siglo XVII dentro del cual se encuentra el testamento de Francisco Hernández transcrito en náhuatl, seguido de su versión en castellano. Otro ejemplo similar está en el MS 10302, que contiene documentos del siglo XVIII recopilados por Lorenzo Boturini, uno de los cuales es el testamento de Diego Lázaro, transcrito en náhuatl y seguido de su traducción al castellano.

En el segundo tipo encontramos los procesos y peticiones a autoridades por parte de individuos o pueblos indígenas, que si bien se integraban a expedientes redactados en su mayoría en castellano, podían incorporar fragmentos textuales en la lengua indígena, por motivos testimoniales.

Un ejemplo está en el expediente manuscrito del siglo XVII ubicado dentro del Archivo Franciscano de la BNM con clasificación AF 49/1066. Se trata de una carta redactada en náhuatl por regidores indígenas y dirigida al "señor tlatoani al[cal]de m[ayor] totepan tlatocatzin", de la provincia de Ávalos.

Como parte del proceso legal, la petición en náhuatl es seguida por su traducción al español, para que fuera comprensible dentro de la esfera administrativa novohispana. El título del manuscrito da una idea de su contenido:

Petizion en lengua mexicana que los Yndios del Pu[ebl]o de Atlaco, Jurisdicion de Zayula, en la prov[inci]a de Avalos, presentaron al Cap[itá]n D[o]n Juan Man[ue]l Serrano y Acuña, Alcalde Mayor de d[ic]ha Prov[inci]a el 9 de Feb[rer] o de 1691, sobre que ynforme al Virrey lo incomodo que resulta el paraje de S[an] Ant[oni]o Tapalpa como Cavezera de Doctrina de los seis Pueblos altos.

En el género legal podrían agregarse otros tipos, relacionados con la esfera administrativa: títulos de tierras, títulos nobiliarios, censos demográficos y catastros. La BNM cuenta con tres manuscritos en lenguas indígenas asociados a estos tipos documentales, que además comparten la particularidad de que su registro es pictográfico; si bien es posible mantener su clasificación dentro 


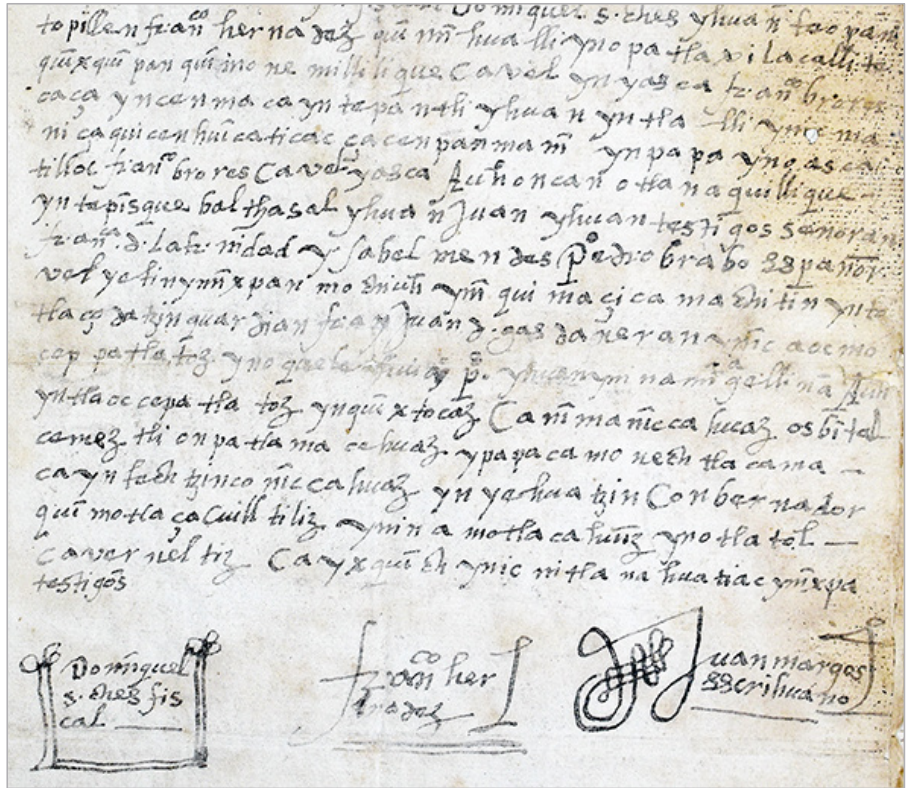

Imagen 10. Testamento de Francisco Hernández, MS 1233, f. 3r.



Imagen 11. Petizion en lengua mexicana de los Yndios de Atlaco, MS AF 49/1066, f. 3r. 
del género legal, se propone agruparlos con un criterio de clasificación por formato escriturario.

Esta propuesta se justifica como una estrategia que busca destacar los pocos manuscritos que conserva la BNM con elementos pictográficos/jeroglíficos de tradición indígena. Es así como el cuarto género documental propuesto es el de los códices pictográficos.

\section{Género de códices pictográficos}

Su característica principal radica en el formato gráfico, relacionado con los sistemas escriturarios y las representaciones pictóricas de tradición indígena. Dentro de este género, los códices Techialoyan constituyen un tipo. Estos documentos fueron producidos entre la segunda mitad del siglo XVII y la primera mitad del XVIII; su elaboración estuvo asociada a los pueblos del centro de México que buscaban el reconocimiento legal de sus tierras comunitarias por parte de las autoridades novohispanas.

Los códices identificados como Techialoyan comparten varios elementos de formato, estilo y contenido que los identifican como un grupo homogéneo; entre los elementos que pueden destacarse encontramos el estilo pictográfico, el estilo literario náhuatl, el molde caligráfico, los tipos de pigmentos, la clase de papel amate y el formato amplio en láminas, así como el propósito con el que fueron concebidos sus contenidos.

El estudio sobre los manuscritos Techialoyan elaborado por Donald Robertson en $1975^{14}$ constituye un punto de consenso para considerar este tipo de códices como un grupo independiente. A partir de entonces se ha impulsado el estudio comparativo al interior de su agrupación, si bien aún falta afinar la metodología de análisis, tal como lo apunta Xavier Noguez: "En la actualidad se conocen cerca de 55 documentos de este grupo. Como se ha mencionado, este corpus es todavía poco conocido debido a su fragmentación, dispersión, falta de publicaciones completas, y a la carencia de uno o varios métodos sintéticos y prácticos de análisis". ${ }^{15}$

\footnotetext{
${ }^{14}$ Robertson, "Techialoyan Manuscripts...", 253-280.

${ }^{15}$ Xavier Noguez, "Códice Techialoyan de San Pedro Tototepec, Municipio de Toluca", en Códice Techialoyan de San Pedro Tototepec (Estado de México), estudio introd. de Xavier Noguez (Zinacantepec: El Colegio Mexiquense, 1999), 12.
} 
El MS 1805 de la BNM, antes conocido como Códice Azcapotzalco, es un fragmento que conforma la tercera parte de un códice Techialoyan que representa al pueblo de Huyxoapan. Los otros dos fragmentos de este códice se encuentran dispersos entre el MS Mexicain 389 de la Bibliothèque Nationale de France y el MS 22070b del British Museum. ${ }^{16}$

Otro tipo de códices pictográficos está vinculado a las genealogías que elaboraban los descendientes de la nobleza indígena, con la intención de reivindicar sus derechos nobiliarios dentro de la estructura colonial. El único ejemplar de este tipo conservado por la BNM es el MS 1809, que tiene una representación pictográfica de don Phelipe Yxitlixochitl, su esposa doña María, sus hijos don Juan Pedro, don Miguel y doña María hija. El manuscrito también contiene la escritura jeroglífica de tradición prehispánica para representar el topónimo de Azcapotzalco y el antropónimo de Yxitlixochitl.

Por último, podemos mencionar el tipo documental relacionado con los censos demográficos y económicos, al igual que los catastros de terrenos. En este rubro encontramos al MS 1497 bis, también conocido como Códice Santa María Asunción, que contiene información relativa a una población de la región de Texcoco. El objetivo de este manuscrito se ubica dentro del contexto de la encomienda en el siglo XVI, tal como proponen Barbara Williams y H. R. Harvey:

Although not explicitly stated in the Asunción, apparently the motive for its production was the need to document the population's ability to pay tribute to the encomendero Gonzalo de Salazar and later to his son, Juan Velázquez de Salazar. [...] Therefore, it appears that the Asunción was drafted initially in the context of tribute reassessment between 1539 and 1544, and then after the pestilence in 1545 it was updated to show the effects of the epidemic. ${ }^{17}$

La relevancia del MS 1497 bis radica no solamente en la información recabada, sino también en los elementos de innovación escrituraria. Aunado a los jeroglíficos antropónimos y topónimos de tradición mesoamericana, el códice incorpora un sistema jeroglífico extendido y adaptado para registrar datos detallados del censo demográfico, tales como el parentesco, el género, la edad y

\footnotetext{
${ }^{16}$ Robertson, "Techialoyan Manuscripts...", 266, 270.

${ }^{17}$ Barbara J. Williams y H. R. Harvey, The Códice de Santa María Asunción. Facsimile and Commentary: Households and Lands in Sixteenth-Century Tepetlaoztoc (Salt Lake City: University of Utah Press, 1997), 2-4.
} 
el estado civil. También desarrolla un sistema gráfico para registrar los terrenos con sus respectivos tamaños y formas, así como las cualidades de explotación del suelo. ${ }^{18}$

Este manuscrito es excepcional, pues muestra cómo incluso dentro del periodo colonial temprano se alcanzó a desarrollar y extender el sistema escriturario de tradición jeroglífica mesoamericana para representar los nuevos elementos del orden novohispano.

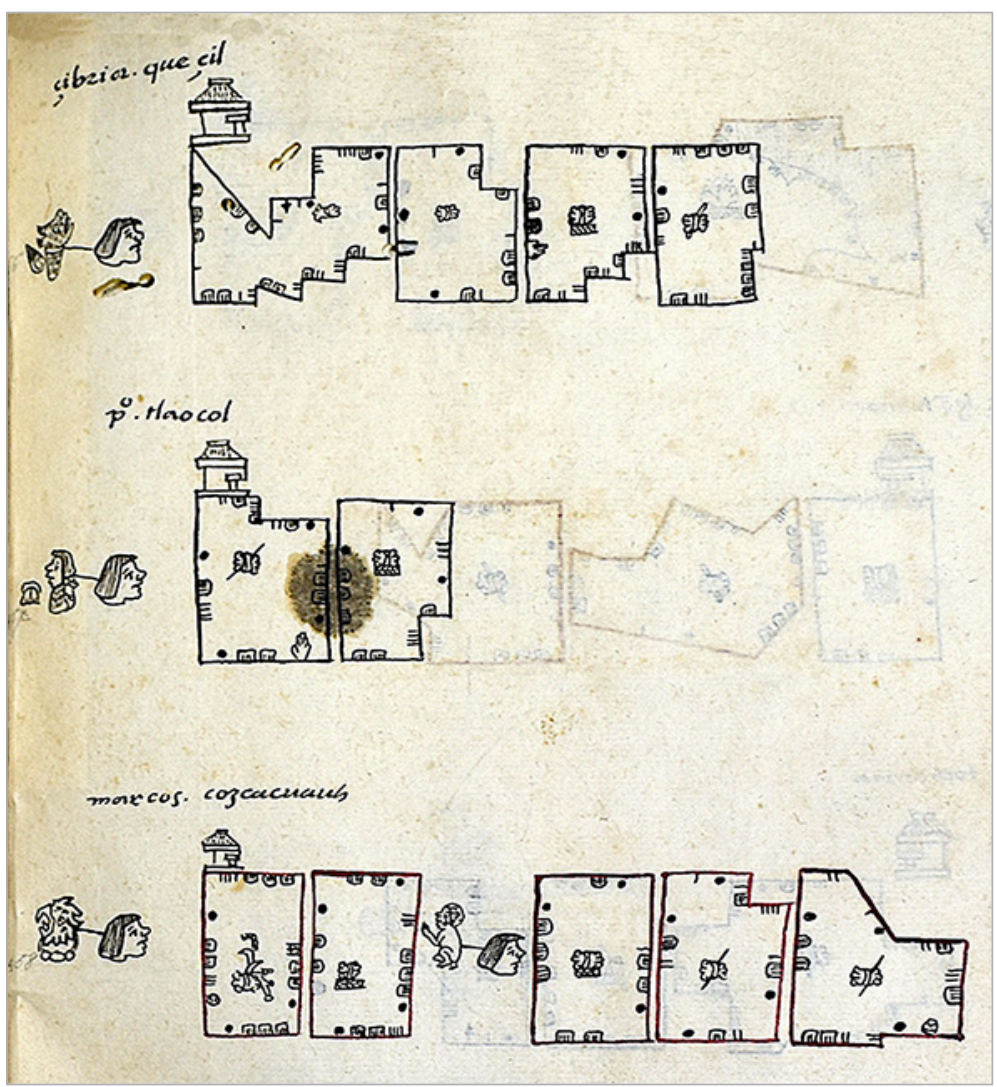

Imagen 12. Códice Santa María Asunción, MS 1497 bis, f. 57r.

${ }_{18}$ Sobre el sistema escriturario jeroglífico en el MS 1497 bis, véase Williams y Harvey, The Códice de Santa María Asunción, 21-53. 




Imagen 13. Códice Techialoyan-Huyxoapan, MS 1805, lámina 2.

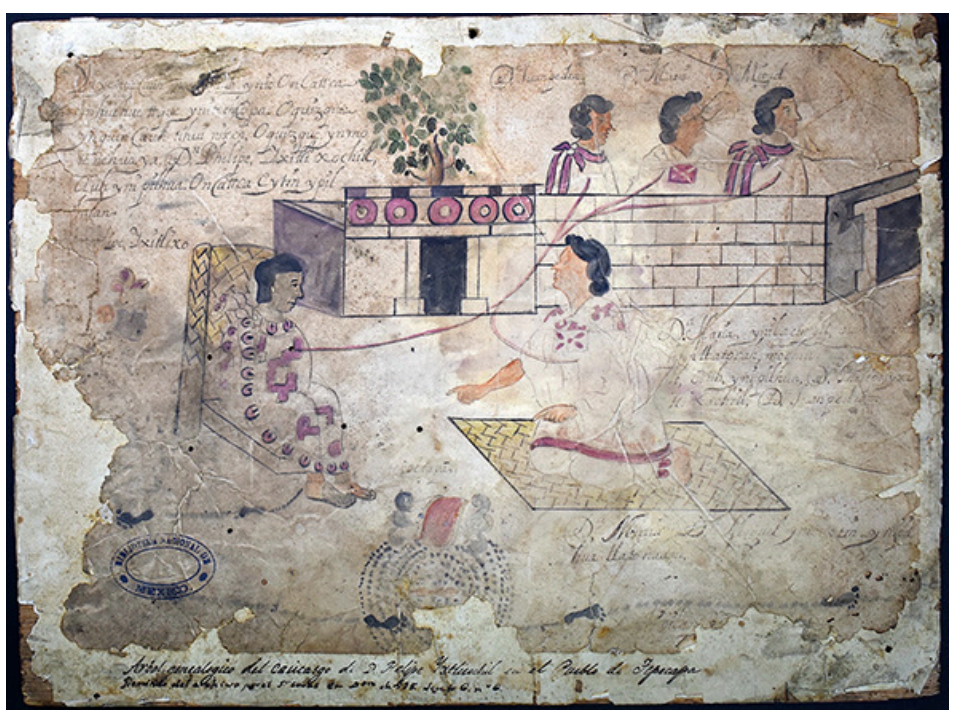

Imagen 14. Genealogía Yxitlixochitl, MS 1809, lámina única. 


\section{Propuesta de clasificación tipológica}

La clasificación tipológica de los manuscritos novohispanos en lenguas indígenas que a continuación se propone fue elaborada a partir del análisis de las especificidades temáticas del corpus conservado en la BNM. La clasificación se estructura en un nivel general y otro particular. En el nivel general se encuentran los géneros documentales que son ejes temáticos que alcanzan una cohesión mediante objetivos discursivos cercanos. En el nivel particular están los tipos documentales que muestran temáticas acotadas y que usualmente están asociados a modelos textuales que orientan su estructura. Además, la división incorpora una clasificación por formato escriturario en el caso de los manuscritos pictográficos, por lo cual puede considerarse una propuesta de clasificación mixta.

\begin{tabular}{|c|c|}
\hline GÉNERO & SUB-GÉNERO \\
\hline Lingüístico & Artes \\
\hline & Vocabularios \\
\hline & Sermones \\
\hline Religioso & Evangeliarios \\
\hline & Santorales \\
\hline & Miscelánea religiosa \\
\hline & Oraciones y rosarios \\
\hline & Testamentos \\
\hline & Procesos y peticiones \\
\hline Lédices pictográficos & Techialoyan \\
\hline & Genealogías \\
\hline & Censos \\
\hline & Catastros \\
\hline
\end{tabular}

Propuesta de clasificación por géneros y tipos documentales de los manuscritos novohispanos en lenguas indígenas de la BNM. Fuente: elaboración propia.

Como mencionamos, la tipología propuesta plantea un nivel general y otro particular, lo cual puede ser útil para la clasificación de obras que contienen diferentes géneros o tipos documentales. Por ejemplo, el MS 1494 pertenece a un solo género, pero a dos tipos, lo cual podría representarse de la siguiente manera: Lingüístico > Artes / Vocabularios. 
Por último, cabe reiterar que la propuesta únicamente consideró manuscritos conservados en la BNM, por lo que la tipología está acotada y no incluye otros géneros documentales que podrían encontrarse en otras bibliotecas. Aún así, la tipología podría ser útil como una orientación en aquellos acervos que también conservan manuscritos novohispanos en lenguas indígenas, e incluso podría ser aplicable si se afinan los tipos documentales de acuerdo con sus propias especificidades.

\section{Conclusiones}

En este artículo se realizó una aproximación a los manuscritos novohispanos en lenguas indígenas resguardados por la Biblioteca Nacional de México. La propuesta de clasificación tipológica consideró la colección como un objeto de estudio, lo cual permitió visualizar el corpus en dos niveles: uno general, en el que se identificaron cuatro géneros documentales; otro particular, que reflejó la diversidad de los tipos documentales.

La intención que conlleva esta propuesta es lograr una mejor comprensión de la colección y de sus respectivas especificidades documentales, lo cual puede constituir un apoyo para la descripción y el análisis de los manuscritos en su dimensión individual.

El ámbito de aplicación de la tipología resulta particularmente útil para los interesados en las obras escritas en lenguas indígenas, puesto que permite una orientación sistematizada dentro del amplio universo documental que abarca el acervo nacional.

Por otra parte, la clasificación tipológica también puede ser útil en el ámbito interno de la propia BNM, puesto que la mayoría de los manuscritos en lenguas indígenas todavía no han sido incorporados al catálogo en línea Nautilo. La tipología propuesta podría ser un apoyo para el proceso de catalogación, así como para el desarrollo de instrumentos de consulta que registren obras en lenguas indígenas.

En este sentido, la aplicación de esta tipología derivaría en una mejor sistematización catalográfica del corpus y, por extensión, en un mejoramiento de los procesos de localización y acceso a los manuscritos que atesora la Biblioteca Nacional de México. 


\section{Referencias}

Garibay Kintana, Ángel María. Historia de la literatura náhuatl. México: Porrúa, 1953. Garibay Kintana, Ángel María. "Manuscritos en lengua náhuatl de la Biblioteca Nacional de México". Boletín de la Biblioteca Nacional de México 17, $2 a$. época, núms. 1-2 (enero-junio de 1966): 5-19.

Glass, John B. "A Census of Middle American Testerian Manuscripts". En Handbook of Middle American Indians. Guide to Ethnohistorical Sources. Vol. 14. Edición de Howard Cline, 281-296. Austin: University of Texas Press, 1975.

Glass, John B. "A Survey of Native Middle American Pictorial Manuscripts". En Handbook of Middle American Indians. Guide to Ethnohistorical Sources. Vol. 14. Edición de Howard Cline, 3-80. Austin: University of Texas Press, 1975.

Hernández Triviño, Ascensión. "La lengua náhuatl en los archivos y bibliotecas de México". En Lenguas y escrituras en los acervos bibliohemerográficos. Experiencias en el estudio de la tradición clásica, indígena y contemporánea. Coordinación de Marina Garone y Salvador Reyes Equiguas, 65-82. México: Universidad Nacional Autónoma de México, 2019.

León-Portilla, Miguel. "El tesoro de los manuscritos indígenas". En La Biblioteca Nacional, triunfo de la República. Coordinación de Vicente Quirarte, 73-81. México: Universidad Nacional Autónoma de México, 2006.

León-Portilla, Miguel, editor. Cantares mexicanos. México: Universidad Nacional Autónoma de México, 2019.

Moreno de los Arcos, Roberto. "Guía de las obras en lenguas indígenas existentes en la Biblioteca Nacional". Boletín de la Biblioteca Nacional de México 17, 2a. época, núms. 1-2 (enero-junio de 1966): 21-117.

Noguez, Xavier. "Códice Techialoyan de San Pedro Tototepec, Municipio de Toluca". En Códice Techialoyan de San Pedro Tototepec (Estado de México). Estudio introductorio de Xavier Noguez, 11-51. Zinacantepec: El Colegio Mexiquense, 1999.

Robertson, Donald. "Techialoyan Manuscripts and Paintings, with a Catalog". En Handbook of Middle American Indians. Guide to Ethnohistorical Sources. Vol. 14. Edición de Howard Cline, 253-280. Austin: University of Texas Press, 1975.

Rosas Xelhuantzi, Tesiu. "Colonización lingüística y subversión decolonial nahua en el siglo XVI". NuestrAmérica 6, núm. 11 (enero-junio de 2018): 72-89.

Williams, Barbara J. y H. R. Harvey. The Códice de Santa María Asunción. Facsimile and Commentary: Households and Lands in Sixteenth-Century Tepetlaoztoc. Salt Lake City: University of Utah Press, 1997. 